\title{
An Iterative Approach for Reconstruction of Arbitrary Sparsely Sampled Magnetic Resonance Images
}

\author{
Hamed Pirsiavash $^{1}$, Mohammad Soleymani ${ }^{2}$, Gholam-Ali Hossein-Zadeh ${ }^{3}$ \\ ${ }^{1}$ Department of electrical engineering, Sharif University of Technology, Tehran, Iran \\ h_pirsiavash@ee.sharif.edu \\ ${ }^{2}$ ECE Dept., Faculty of Eng., University of Tehran, Tehran 14395-515, Iran \\ m.soleymani@ece.ut.ac.ir \\ ${ }^{3}$ ECE Dept., Faculty of Eng., University of Tehran, Tehran 14395-515, Iran \\ ghzadeh@ut.ac.ir
}

\begin{abstract}
In many fast MR imaging techniques, $K$-space is sampled sparsely in order to gain a fast traverse of $K$-space. These techniques use non-Cartesian sampling trajectories like radial, zigzag, and spiral. In the reconstruction procedure, usually interpolation methods are used to obtain missing samples on a regular grid. In this paper, we propose an iterative method for image reconstruction which uses the black marginal area of the image. The proposed iterative solution offers a great enhancement in the quality of the reconstructed image in comparison with conventional algorithms like zero filling and neural network. This method is applied on MRI data and its improved performance over other methods is demonstrated.
\end{abstract}

\section{Introduction}

Magnetic resonance imaging is a widely used modality of medical imaging which is based on the electromagnetic fields of resonated atomic nuclei. By changing spatial and temporal factors in magnetic field intensity, a signal is induced in receiver coil. The samples of this signal are Fourier transform of tissue image. These samples are located in the frequency domain, namely K-space [1, 2].

To reduce the imaging time, k-space data is obtained via new and fast imaging techniques. These fast approaches are applicable in capturing brain dynamic activities through functional MRI.
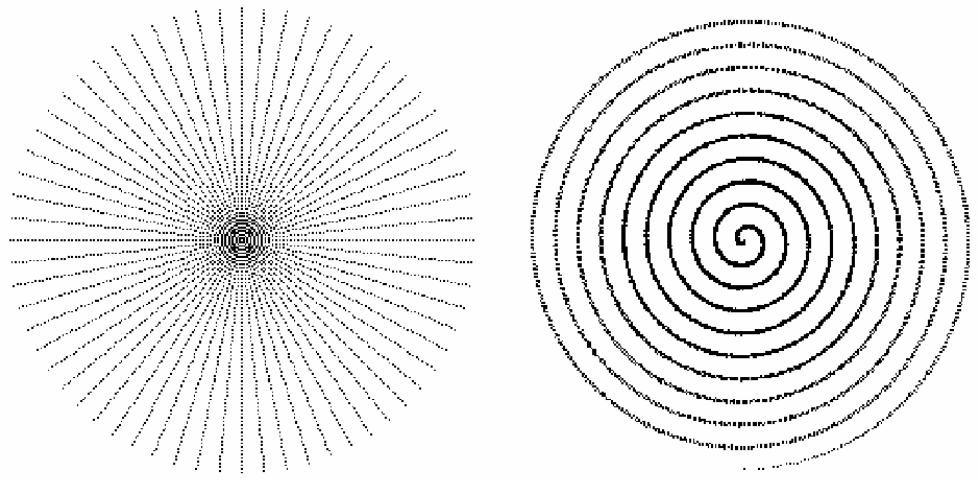

Figure1. Two sampling trajectories of the k-space (left: radial, right: spiral).

Spiral and radial trajectories used to sample k-space are shown in figure 1. Radial, Cartesian and zigzag trajectories are made by straight lines and usually pass through k-space center [3]. Radial sampling is less sensitive to motion artifacts and robust against fast decay of signal [4]. Although the main advantage of the Cartesian sampling is simplicity in 
reconstruction through inverse FFT, this sampling process is usually time consuming. On the other hand, non-Cartesian trajectories offer lower imaging time in the expense of higher computational complexity needed for image reconstruction from sparsely sampled k-space $[1,2]$.

The reconstruction techniques are described in section 2 . The proposed algorithm comes in section 3 and implementation results are illustrated in section 4. Finally, the conclusion is discussed.
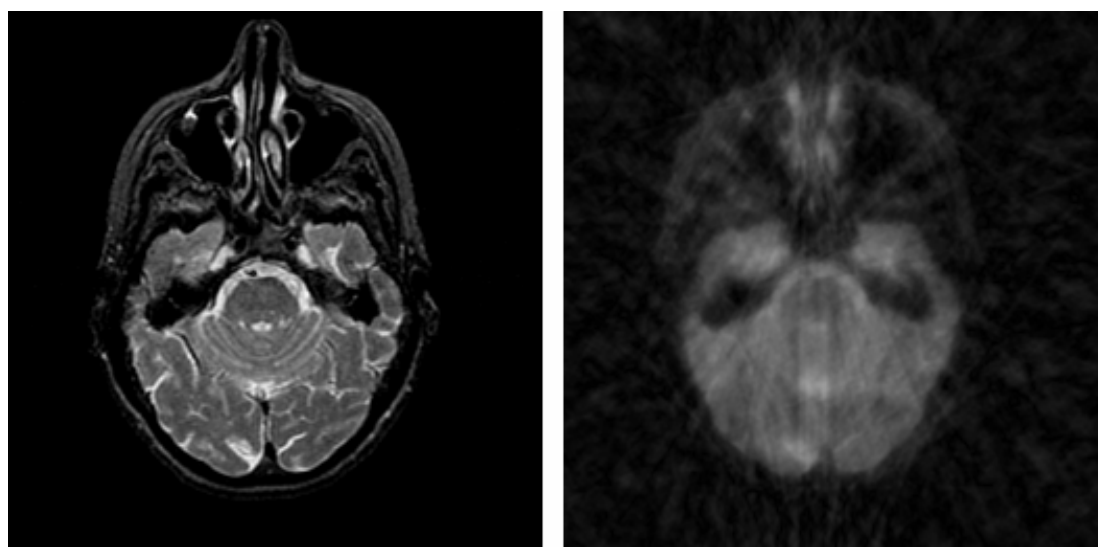

Figure 2.Left: Original image; Right: Reconstructed image using zero filling method

\section{Reconstruction techniques from sparsely sampled K-space}

The missing samples are replaced by zero in zero-filled k-space. By this strategy, although the reconstruction procedure is fast, the image quality will be poor. One example of an MR image reconstructed by this technique is shown in figure 2. The linear and bilinear interpolations are other strategies of calculating missed samples [2]. Bayesian estimation is also used as an alternate technique for reconstruction of sparsely sampled k-space [1]. In the literature, an iterative approach is also used for reconstruction which avoids the problem of degrading image quality [5]. Reczko and his colleagues in [4] introduced a machine learning approach via artificial neural networks in order to compute missed samples.

\section{Iterative method}

Most MR images have black areas around their contents. This fact is the key point of this new algorithm. In complete Cartesian sampling, the DFT and IDFT equations relate k-space samples and image pixels but in sparsely sampled $\mathrm{k}$-space the equations are ill-posed. Using samples of k-space and some known pixels which are black around image, some numerical solutions are available to recover missed samples. This paper proposed a fast and simple numerical solution for these equations using an alternation between the k-space and the spatial domain. The diagram of the proposed algorithm is shown in figure 3.

In the first stage, one image is reconstructed by zero filling method then a bounding box for the image contents is extracted using Sobel edge detection method. In the zero-filled image surrounding pixels are not purely black; hence, we should set a periphery for the image and force the surrounding pixels to be zero. In the next step, modified k-space values are computed with FFT that results in a regular grid. In this grid the values of some pixels that belong to the sampling trajectories are replaced with the original measured data, and the 
image is reconstructed again. This process is repeated in a conditional loop to reach an enhanced MR image. The loop end condition will be met when the mean square difference between outputs of two successive iterations become less than a predefined threshold or the iteration number reaches the maximum value. To improve iteration result, bounding box should be updated after each stage. Putting edge detector block in the loop makes a great enhancement over results because it increases the number of known parameters during iterations.

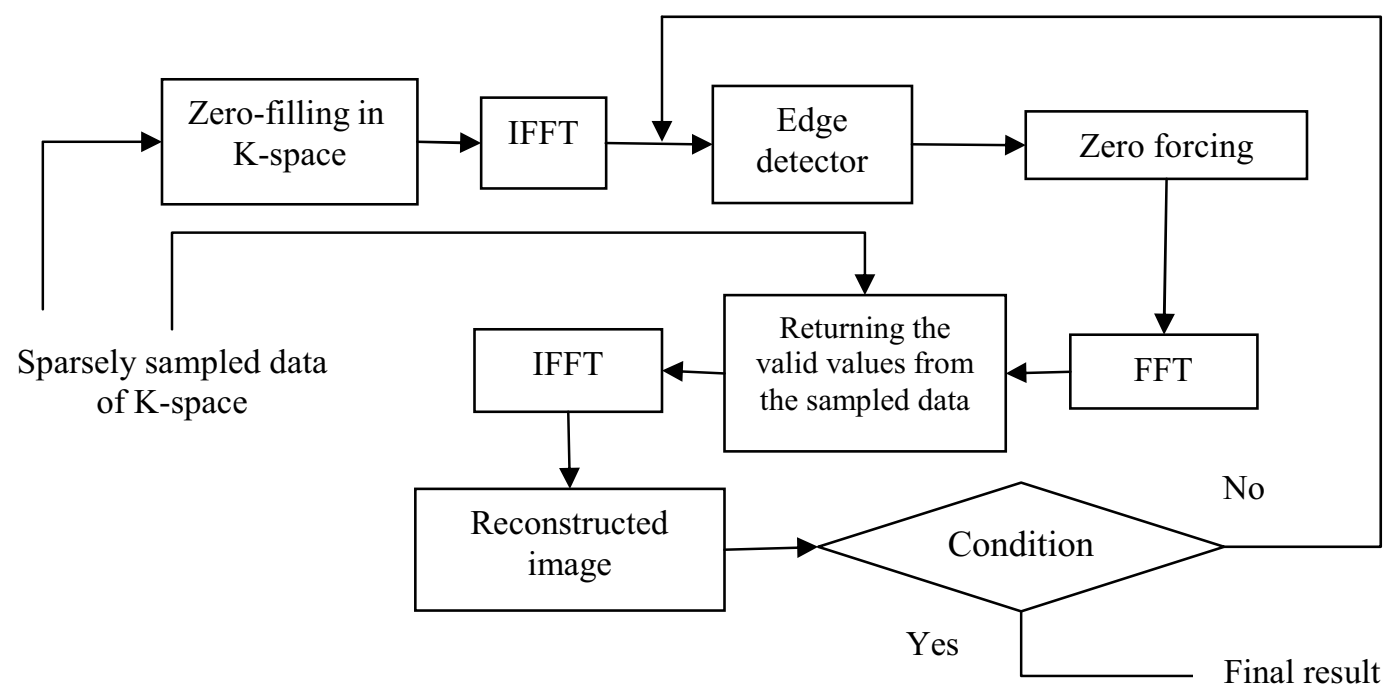

Figure 3.Block diagram of the proposed algorithm

\section{Implementation and results}

Zero filling, neural networks [4] and our proposed method are implemented in MATLAB platform and applied to 117 randomly selected MR images from database of Harvard human brain atlas ("http://www.med.harvard.edu/AANLIB/"). In these implementations, image quality is measured using two different quantitative parameters. The first one is the peak signal to noise ratio and the other named RMS-SNR proposed by Dologlou et al. [6] is defined below.

image_resid $=$ image_orig $-\frac{\sum \sum \text { image_recon }(i, j) \cdot \text { image_orig }(i, j)}{\sum \sum \text { image_recon }(i, j) \cdot \operatorname{image} \_r e c o n(i, j)} \cdot$ image_recon
$R M S_{-} S N R=10 \cdot \log _{10} \frac{\sum \sum \text { image_orig }(i, j) \cdot \operatorname{image} \_ \text {orig }(i, j)}{\sum \sum \text { image_resid }(i, j) \cdot \operatorname{image} \_r e s i d(i, j)}$

The implemented neural network is a multi layer perceptron which has 48 inputs, 20 hidden and 2 output neurons. The training set is selected from six images sampled by both radial and spiral trajectories. The neural network inputs are both real and imaginary part of the neighboring point values in K-space and the outputs contain two parts of the center point complex value. 
Table 1.Simulation results of 117 selected images from Harvard database.

\begin{tabular}{|c|c|c|c|c|c|c|c|c|}
\hline File name & $\begin{array}{c}\text { Sampling } \\
\text { Trajectory }\end{array}$ & \multirow{2}{*}{$\begin{array}{c}\text { Sampling } \\
\text { density }\end{array}$} & \multicolumn{2}{|c|}{ Zero Filling } & \multicolumn{2}{c|}{ Neural Network } & \multicolumn{2}{c|}{ Iterative Algorithm } \\
\cline { 4 - 9 } & & & $\begin{array}{c}\text { PSNR } \\
(\mathrm{db})\end{array}$ & $\begin{array}{c}\text { RMS- } \\
\text { SNR(db) }\end{array}$ & $\begin{array}{c}\text { PSNR } \\
(\mathrm{db})\end{array}$ & $\begin{array}{c}\text { RMS- } \\
\text { SNR(db) }\end{array}$ & $\begin{array}{c}\text { PSNR } \\
(\mathrm{db})\end{array}$ & $\begin{array}{c}\text { RMS- } \\
\text { SNR(db) }\end{array}$ \\
\hline Tc1 & Spiral_30 & 0.3656 & 10.56 & 2.98 & 19.24 & 8.52 & 25.33 & 17.73 \\
\hline Tc1 & Radial_128 & 0.1161 & 13.95 & 6.36 & -- & -- & 24.97 & 17.37 \\
\hline Tc1 & Radial_128 & 0.2191 & 23.14 & 15.53 & 24.2 & 16.82 & 32.45 & 24.84 \\
\hline T14 & Spiral_30 & 0.3656 & 14.52 & 3.71 & 21.45 & 8.72 & 37.67 & 26.85 \\
\hline T14 & Radial_128 & 0.2191 & 24.87 & 14.05 & 26.95 & 18.92 & 41.87 & 31.05 \\
\hline Dg1 & Spiral_30 & 0.3656 & 16.87 & 3.54 & -- & -- & 46.08 & 32.74 \\
\hline Dg1 & Radial_128 & 0.2191 & 24.12 & 10.77 & 25.68 & 13.12 & 43.02 & 29.67 \\
\hline 038 & Spiral_30 & 0.3656 & 15.77 & 3.84 & -- & -- & 42.16 & 30.21 \\
\hline 038 & Radial_128 & 0.2191 & 24.19 & 12.24 & 27.53 & 18.52 & 42.11 & 30.16 \\
\hline $\begin{array}{c}\text { Database } \\
\text { mean }\end{array}$ & Spiral_30 & 0.3656 & $\mathbf{1 4 . 1 6}$ & $\mathbf{3 . 4 8}$ & $\mathbf{1 9 . 7 3}$ & $\mathbf{1 0 . 8 6}$ & $\mathbf{3 8 . 2}$ & $\mathbf{2 7 . 5 0}$ \\
\hline $\begin{array}{c}\text { Database } \\
\text { mean }\end{array}$ & Spiral_15 & 0.1839 & $\mathbf{1 3 . 1 4}$ & $\mathbf{2 . 7 0}$ & -- & -- & $\mathbf{2 1 . 7 5}$ & $\mathbf{1 1 . 1 4}$ \\
\hline $\begin{array}{c}\text { Database } \\
\text { mean }\end{array}$ & Radial_128 & 0.2191 & $\mathbf{2 4 . 4 3}$ & $\mathbf{1 3 . 7 3}$ & $\mathbf{2 9 . 8 4}$ & $\mathbf{1 6 . 4 5}$ & $\mathbf{4 3 . 7 0}$ & $\mathbf{3 3 . 0 1}$ \\
\hline $\begin{array}{c}\text { Database } \\
\text { mean }\end{array}$ & Radial_64 & 0.1131 & $\mathbf{2 1 . 4 4}$ & $\mathbf{1 0 . 7 5}$ & -- & -- & $\mathbf{3 4 . 2 4}$ & $\mathbf{2 3 . 5 4}$ \\
\hline $\begin{array}{c}\text { Database } \\
\text { mean }\end{array}$ & Radial_32 & 0.0574 & $\mathbf{1 9 . 5 2}$ & $\mathbf{8 . 8 3}$ & -- & -- & $\mathbf{2 7 . 7 1}$ & $\mathbf{1 7 . 0 2}$ \\
\hline
\end{tabular}

In the proposed method, the maximum number of iterations is set to 100 in order to reduce the process time. In most cases, number of iterations becomes less than the maximum limit. Because of high computational complexity of the Sobel edge detector operator, the bounding box is found once in three successive iterations.

The results for some selected images used in [4] and the overall quality measures have been presented in table 1. There are some blank cells in table because running the neural network method is time consuming and not necessary for all cases. Mean overall results of the whole test set comes in five last rows. Some result images for sampling trajectories and two methods have been shown in figures 4 and 5. Figure 6 shows the improving curve of the PSNR versus number of iterations for one selected image sampled using 30 spiral trajectories. It can be seen apparently that edge detection process in 38th iteration increased the number of known parameters and enhanced the saturated PSNR curve.
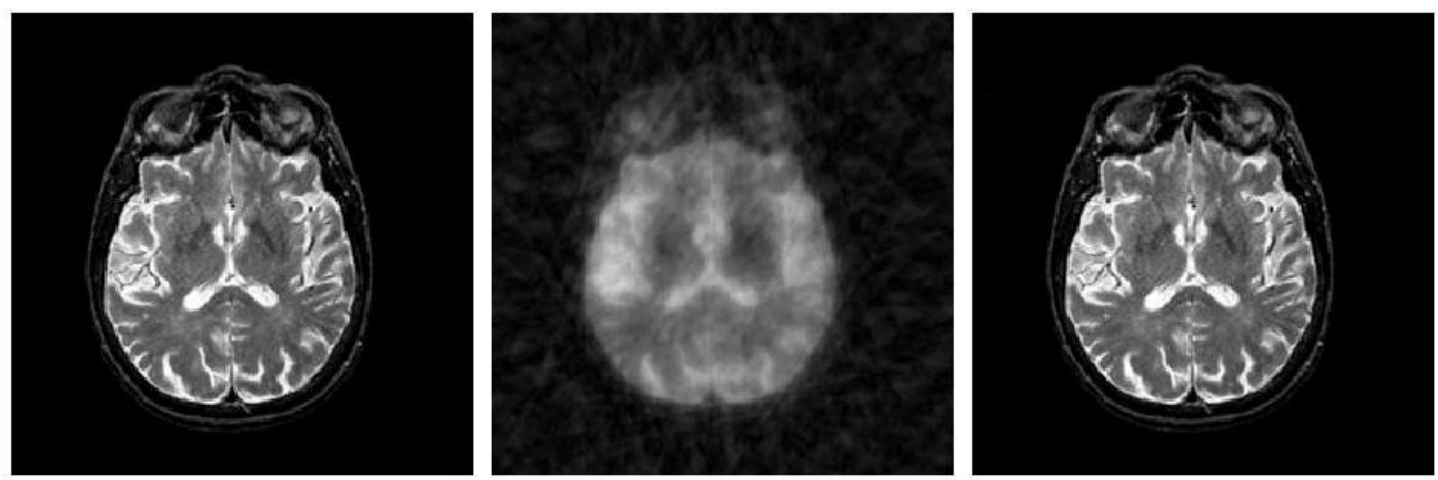

Figure 4.An example sampled using 32 radial trajectories. (From left to right: original image, zero-filled image, and iterative reconstructed image) 

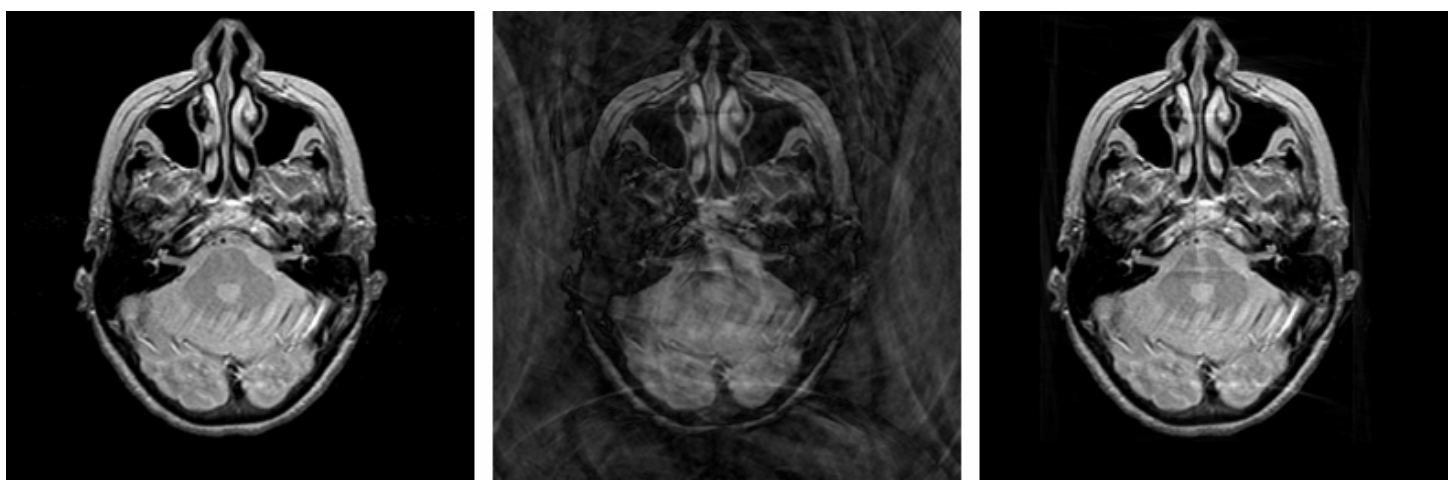

Figure 5.An example sampled using 30 spiral trajectories. (From left to right: original image, zero-filled image, and iterative reconstructed image)

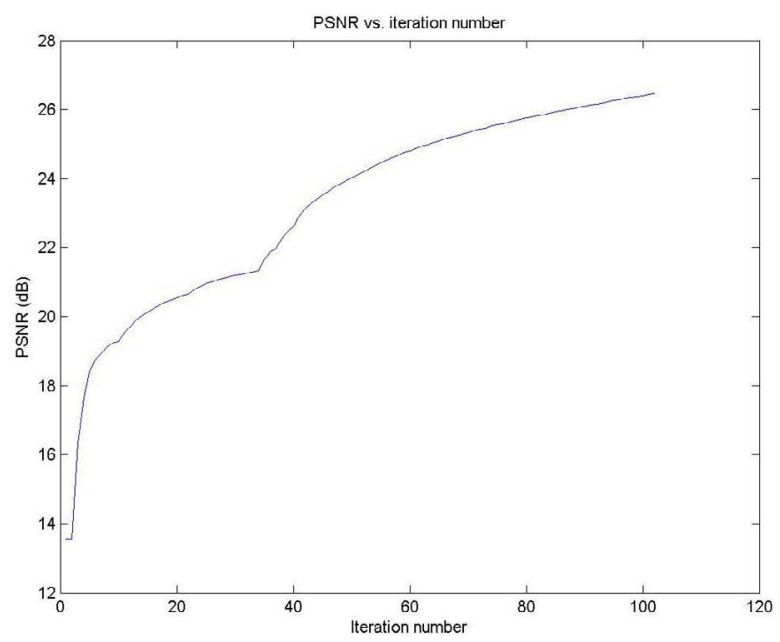

Figure 6. PSNR vs. number of iterations for an example image sampled using 30 spiral trajectories

\section{Conclusion}

A novel and model free iterative method is presented for reconstruction of sparsely sampled MR images. This algorithm has been implemented and tested successfully on several MR images. The results show that in most MR images, we can use the marginal black area and improve the reconstructed image quality. The radial sampling method in comparison with the spiral one has higher density near the center of K-space; hence, zero-filled and iteratively reconstructed images for this sampling trajectory have better quality.

\section{References}

[1] Zhi-Pei Liang and P.C. Lauterbur, "Principles of Magnetic Resonance Imaging: A Signal Processing Perspective", IEEE press, 1999.

[2] H. Yan, "Signal processing for magnetic resonance imaging and spectroscopy", Marcel Dekker Inc., 2002.

[3] H. Yan and M. Braun, "Image reconstruction from fourier domain data sampled along zig-zag trajectory," Magnetic Resonance in Medicine, vol 36, 834-846, 1996. 
[4] M. Reczko, D.A. Karras, B.G. Mertzios, D. Graveron-Demilly, and D. van Ormondt, "Improved MR image reconstruction from sparsely sampled scans based on neural networks", Pattern Recognition Letters 22, 35-46, 2001.

[5] B. Desplanques, J. Cornelis, E. Achten, R. Van de walle, and I. Leahieu, "Iterative reconstruction of magnetic resonance images from arbitrary samples in k-space", IEEE transactions on nuclear science, VOL 49, NO 5,2002. [6] I. Dologlou, F.T.A.W.Wajer, M.Funderer, D. van Ormondt, "Spiral MRI scan-time reduction through nonuniform angular distribution of interleaves and multichannel SVD interpolation", proceedings of ISMRM Fourth Meeting, New York, 1996. 